\title{
IMAGE
}

\section{Insights Figure for Anti-thymic stromal lymphopoietin antibody suppresses airway remodeling in asthma through reduction of MMP and $\mathrm{CTGF}$}

Sheng-Chieh Lin $^{1,2,3}$, Hsiu-Chu Chou ${ }^{4}$, Chung-Ming Chen $\mathbb{D}^{2,5}$ and Bor-Luen Chiang ${ }^{3}$

Pediatric Research (2019) 86:285; https://doi.org/10.1038/s41390-019-0457-x

Schematic diagram showing anti-TSLP antibody actions in airway remodeling in asthma. As depicted, anti-TSLP antibody inhibits OVA-induced airway remodeling. $\downarrow$ indicate activation or induction; $\perp$ indicates inhibition or blockade.

\section{REFERENCE}

Lin, S.-C., Chen, H.-C., Chen, C.-M., Chiang, B.-L. Anti-thymic stromal lymphopoietin antibody suppresses airway remodeling in asthma through reduction of MMP and CTGF. Pediatr Res. (2018). https://doi.org/10.1038/s41390-018-0239-x. [Epub ahead of print].

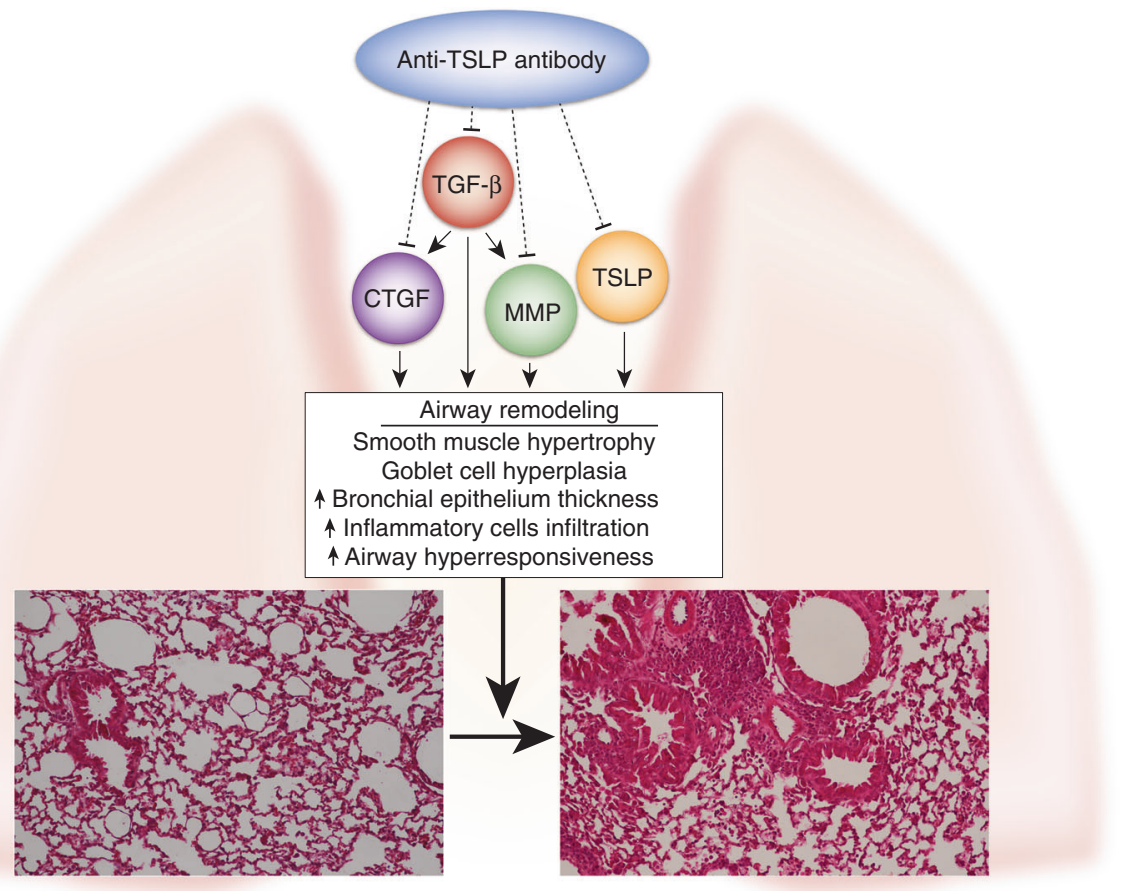

'Department of Pediatrics, Shuang Ho Hospital, Taipei Medical University, Taipei, Taiwan; ${ }^{2}$ Department of Pediatrics, School of Medicine, College of Medicine, Taipei Medical University, Taipei, Taiwan; ${ }^{3}$ Graduate Institute of Clinical Medicine, College of Medicine, National Taiwan University, Taipei, Taiwan; ${ }^{4}$ Department of Anatomy and Cell Biology, School of Medicine, College of Medicine, Taipei Medical University, Taipei, Taiwan and ${ }^{5}$ Department of Pediatrics, Taipei Medical University Hospital, Taipei, Taiwan Correspondence: Chung-Ming Chen (cmchen@tmu.edu.tw) or Bor-Luen Chiang (gicmbor@ntu.edu.tw)

Received: 17 May 2019 Accepted: 29 May 2019

Published online: 13 June 2019 